\title{
Contexts, Pleasures and Preferences: Girls Playing Computer Games
}

\author{
Diane Carr \\ Institute of Education \\ London Knowledge Lab \\ 23-29 Emerald Street \\ London, WC1N 3QS \\ d.carr@ioe.ac.uk
}

\begin{abstract}
In this paper, issues of girls and their gaming preferences are explored through observations of computer games sessions at an all-girl state school. What emerged is that preferences are alterable, and site specific. Gaming selections relate to the attributes of particular games - but they also depend on a player's recognition of these attributes and the pleasures they entail. Players accumulate these competencies according to the patterns of access and peer culture they encounter. Thus preferences are an assemblage, made up of past experiences, and subject to situation and context. The constituents of preference, such as access, are certainly shaped by gender. As a result, gaming preferences may manifest along gendered lines. It is not difficult to generate data indicating that gendered tastes exist, but it is short sighted to divorce these outcomes from the various practices that contribute to their formation.
\end{abstract}

\section{Keywords}

computer games, gender, preference, access

Women and girls in the West have reputedly refused to embrace computer games with the same zeal as their male peers. Variously motivated theorists from a range of disciplines have investigated the reasons behind the statistics, and proposed remedies for the situation. One thing these diverse efforts $[1,3,5,6,7]$ have tended to share is an interest in girls and their gaming preferences.

Here the question of girls and their preferences is discussed with reference to observations of a computer games club at a single sex state school in South London. Play observations were combined with video documentation, interviews and questionnaires in order to investigate these girls' gaming tastes. These observations suggest that when considering games, gender and preference, it is productive to employ a holistic and mobile or dynamic account of tastes and inclination - one that implicitly incorporates existing work within media and cultural studies on active and media literate audiences and their 'reading histories' [2,4]. This is important, because what became apparent over the course of a school term is that preferences are not static, nor do they emerge in a vacuum. The problem with ascribing particular preferences directly or solely to

Proceedings of DiGRA 2005 Conference: Changing Views - Worlds in Play.

(C) 2005 Authors \& Digital Games Research Association DiGRA. Personal and educational classroom use of this paper is allowed, commercial use requires specific permission from the author. 
player gender is that it implicitly divorces gaming tastes from the economic, social and cultural forces that fuel and inform gaming practices. It is worth noting that here, again, the question of games and gender focuses on the feminine. Games, gaming and masculinity is a topic calling for investigation, but one that is outside of the scope of this particular piece of research. The discussion that follows is quite tightly framed (see 'acknowledgements') with the result that important issues, such as the historical and commercially motivated construction of the male player as the 'norm' (and associated practices of female disenfranchisement), have been omitted.

Generally speaking, digital game developers, publishers, retailers and their high profile marketing campaigns have addressed a male audience. One unsurprising result of this is that this sector of the potential gaming market has swelled, while others have remained relatively untapped. There are signs that things are changing, and it is feasible that as games continue to shift out of the sub-cultural margins and into the mainstream, distinctions other than that of gender may become more pertinent; that diversity will be further accommodated, and patterns of participation may alter.

It was certainly the case that for the 913 -year old girls in the gaming club, computer and video games were a routine part of their lives. They were all casual, rather than hard-core gamers, in that they played for a couple of hours a week, rather than hours per day (although there were signs that at least one of the players desired much greater levels of access). The girls talked with their friends about games, played at home and when they visited family, and sought out information online or in gaming magazines occasionally. When interviewed the club members referred to games as a regular, pleasurable yet otherwise unremarkable part of their younger childhood. One of the girls, in fact, was jubilant that her dad had just promised her a new Game Boy as a reward for good grades. We issued questionnaires to 55 of the girls' classmates, and found similar results. There were certainly varying degrees of access, but most played digital games of one sort or another on a fairly regular basis.

Of course there are multiple factors and discourses active in contemporary culture and in the lives of male and female players, impacting on how games are regarded, and how players position themselves in relation to gaming culture. In the play sessions observed during this research, factors including humor, music, the attractiveness of certain male avatars, or the appetite for all things Harry Potter, were integral to the choices and preferences demonstrated. These 13 year-old female players would also discuss the merits of the different platforms, and particular games, not just as players, but as canny, proto-contenders in consumer culture. This discourse cannot be divorced from wider gaming culture (where it links with notions of cultish expertise), nor can it be entirely separated from considerations of gender, age, race or class.

\section{PLAY AND SITUATED PREFERENCE}

Most of the participants who joined the computer games club were suggested by the school, a few girls heard about the club and volunteered. The group quickly settled at 9 members, with a few regular spectator/visitors and an ever-expanding waiting list. Once the club was up and running it became necessary to limit entry in order to prevent overcrowding. The club ran on Tuesday lunchtimes for the duration of a school term. There is no pretence in this discussion that this is a particularly 'natural' situation - the focus here is on the constituents of preference in a particular situation, and the links between access, context and preference, so the fact that this is an arranged rather than 'found' scenario is not felt to be an issue. One or two adults were 
generally present, and would occasionally help out with technical issues or even join in a games session - but they did not orchestrate player groupings. The author did not participate in actual game-play but rather observed and video-documented the sessions.

At the start of the term, and again at the end, the girls completed questionnaires on which they were asked to list their favorite games. On both questionnaires the girls listed a wide variety of games and genres: fighting and racing games were mentioned repeatedly, games from the Grand Theft Auto series were popular, as were action adventure games and The Sims. In the context of this paper, however, what is significant is that the girls listed a total of 26 games on the later questionnaire, and this included 16 new games, 9 of which they had played during the sessions. The girls' new favorites included named titles (Midtown Madness 3, Jak and Daxter), but also entirely new genres ('fighting games'). The initial preferences expressed on the first questionnaire presumably relate to games that the girls had previously accessed. The alterations shown in the later responses could reflect a number of variables - the girls being in a different mood, for instance. These shifts, however, also indicate that simply offering these users alternative games in a new context was sufficient to generate changes in their stated tastes. What this suggests is that when investigating gaming inclinations the relationship between previous access and expressed preferences should not be overlooked.

Certain site specifics were implicated in the preferences demonstrated by these users. These included the layout of the room, as well as timing, territory and hardware (who's turn it is, who got there first, etc.). Obviously the games supplied were also a factor in the girls' choices (see table 1), as was the particular type of play offered by different games, and the allure of popular trans-media franchises. We attempted to supply a mixed bag of popular games, but with a limited budget we were reliant to some degree on the games that we had to hand. The games were piled on a table in the centre of the room and the girls were free to help themselves. If a girl did not have a particular game in mind she might rifle through the available titles and pick out one based on its packaging, but if the game-play did not appeal, it would soon be discarded.

\begin{tabular}{|r|r|r|}
\hline XBox & $\begin{array}{r}\text { Halo, Midtown Madness 3, Terminator 3, } \\
\text { PS2 }\end{array}$ & $\begin{array}{c}\text { Jak and Daxter, Tomb Raider 4, Tony Hawks Pro Skater } \\
\text { 4, Abe's Oddysee, Oni, Primal, Soul Reaver 2, Time Splitters 2, XIII, Enter The Matrix }\end{array}$ \\
\hline PC & $\begin{array}{c}\text { Deus Ex, Dino Crisis, Lord of the Rings; The } \\
\text { Fellowship of the Ring, Neverwinter Nights, Warcraft 3, Tomb Raider 2, Baldur's Gate, The Sims, } \\
\text { Harry Potter and the Philosopher's Stone, Tony Hawks Pro Skater 3, Black \& White, Sid Meier's } \\
\text { Civilization III, HalfLife, Harry Potter and the Prisoner of Azkaban }\end{array}$ \\
\hline
\end{tabular}

Table 1: Games supplied during the term.

While most of these games were picked and played at some point, certain games were picked with notable regularity. There was a Playstation 2 and an Xbox, and 6 PCs in the room. The consoles were near the door, and offered 2-player games. For this reason, the area around the consoles was the loudest and the most social part of the room and thus, in this specific instance the consoles were less conducive to private or contemplative gaming than the PCs. As an outcome, the most popular games on the consoles were the ones that offered instant gratification, pace, tricks, humor, dual-play and turn taking, rather than games that were orientated towards narrative or that demanded sophisticated tactics. This meant that Tony Hawks Pro Skater 4 (a skateboarding game), Midtown Madness 3 (a comical driving game) and Dead or Alive 3 (a 
fighting game) were consistently selected, and played for longest on the consoles. The stunts, options, character selections and special moves in these games were accessed in an experimental, playful manner. Skills acquisition at the consoles did occur, yet it did not look particularly systematic - one player stated that she liked Midtown Madness 'because I crashed into everything'- but another girl said that she enjoyed learning new tricks in Tony Hawks, while the girls who liked Dead or Alive 3 appreciated that there were 'loads of new moves to learn'. One player remarked that 'this game was good cos they had really good graphics and I'm always winning'. The girls were happy to compete in Dead or Alive- the players occasionally sang or danced when they won a round - but instances of coaching were also frequent.

The PCs, on the other hand, were placed in a quieter section of the room and as an apparent consequence different genres were preferred. Here the girls generally put on headphones to settle in for 30 minutes of solo gaming. Onlookers were tolerated but interruptions were not welcome and there was little or no turn taking. Different games were tried out, but the most consistently popular were The Sims, the two Harry Potter games and the Lord of the Rings games. These action adventure games reward a player's investment of time with characterization, sequential missions or storytelling. The Harry Potter games are 'fun because [they] are just like the movie/book and you get to learn the spells'. The Sims offers incremental complexity and the chance to take control - as one player explained: 'I could make their lives enjoyable or miserable'.

So, the division of the room into noisier and quieter areas, and into sociable or more private zones, combined with the styles of play (instantaneous, or more contemplative) that various games offer. In short, the preferences demonstrated by the girls were shaped by the context of play, in combination with the styles of play that various games offer, and the player's awareness of such offers. In each instance, the ability of the girls to recognize and actualize the pleasures promised by different games was enabled by their being literate in the forms of play available, and the kinds of experiences potentially on offer.

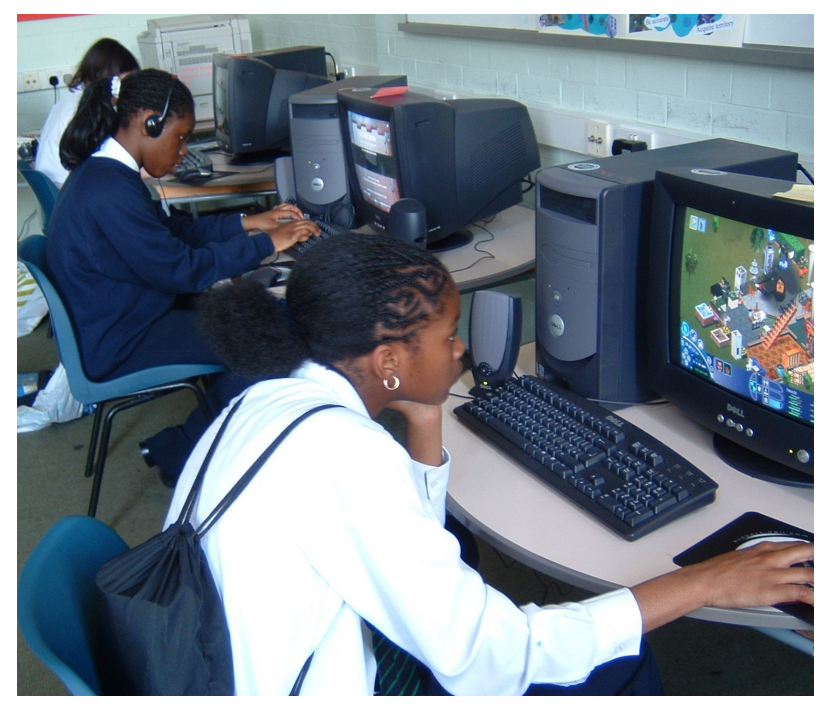

Figure 1: Players at the PCs 


\section{TEXT AND REPRESENTATION}

As facilitators we made a concerted effort to include as many female avatars as possible, so a reasonable proportion of the games we supplied featured female leads - and showed images of the heroine on their packaging. These included Oni, Primal, Enter The Matrix, Dino Crisis, Tomb Raider IV and Tomb Raider II (while in the RPGs Baldur's Gate and Neverwinter Nights the player has the option to construct a female lead character). For some women players the manner in which female avatars are included, excluded or depicted is certainly an issue (see www.womengamers.com for a discussion of 'digital women'). These girls, however, showed no noticeable, additional interest in these games. In fact we were impressed by the degree of ambivalence they inspired. Perhaps this was because of the girls' age, or because they were in a primarily female environment, or because they were in public. Whatever the reason, this disinterestedness is enough to suggest that 13-year-old girls playing games at a single sex school do not regard a female avatar as a particular bonus. When given the option to play as either a male or a female character, the girls would either show no preference and playfully switch between the options (as with Dead or Alive 3), or they might actually prefer to play with/as a male avatar despite having the option of a female - as was consistently the case with Tony Hawks Pro Skater 4.

We distributed a questionnaire to 55 of the club members' fellow students (same year, same school) in order to contextualize our observations of the gaming sessions. It included questions relating to games, access and tastes. For instance, the girls were asked 'If you could create a computer game or video game character, what would they be like?' Nearly $60 \%$ of those who answered this question did not specify a gender for their character at all - they instead listed nonspecific characteristics ('joyous', 'funny', 'loud, adventurous' and 'independent') or included the choice of male or female. Around $25 \%$ of the respondents wanted to play with funny or attractive male characters ('strong, buff male, muscley' or 'tall, strong and rich with good taste and style', 'muscley, dark, good looking', 'six pack, single plaits in hair'). The smaller remainder specified that they would create a female character. Interestingly, it was girls with the highest degree of access (i.e. girls with a games console at home that they considered 'their own') who were the most likely to specify a gender for their avatar, plus they were slightly more likely to designate it male. In other words, the allocation of a particular gender or maleness to an avatar might not reflect the preference of a player, as much as it reflects that player's familiarity with console game conventions.

When asked to list the attributes of the best games at the gaming sessions, the girls gave the following responses: being in control (especially of The Sims), and having good music and graphics. A game should have choice and variety; it should be unpredictable. There should be action and a range of characters, and the possibility of interesting missions and tasks. Magic and adventure were listed as desirable. Plus, it was noted, a game should be easy to learn, yet hard to play (but not too hard!). In order to demonstrate, of course, that there was anything particularly feminine about these preferences it would be necessary to contrast them with the preferences of these players' male peers (peers in terms of age, and also in terms of their being casual rather than hardcore gamers).

The presence of these desired characteristics, however, was no guarantee of popularity. Other factors still determine if a player will become aware of an unfamiliar game's offers and potentials - or not. For instance, none of the club members showed any interest in playing Buffy 
The Vampire Slayer until the penultimate week of term, when a few of the girls arrived early and found the sessions' facilitators playing it on the Xbox. What they saw of Buffy's game-play was sufficient to spark their interest. Then, by passing around the controls, calling out instructions to each other, and shrieking at the 'scary bits', the girls managed to convert it into a rowdy and sociable group activity. Thus several factors were complicit in their late predilection for Buffy. First, the game only became interesting after the girls had seen others engrossed by it. Secondly, after a term with Tony Hawks and Dead or Alive 3, the girls had become adept at generating their own preferred form of group play.

We had also supplied a number of RPGs for the PCs including Baldur's Gate and Neverwinter Nights. These games involve creating a central character, using spells and potions, and exploring a vast fantasy world. As noted, these players stated preferences for variation in character, magic, missions and adventuring, yet none chose to play these games. The players had no prior experience with the genre, and this appears to have played a part in their disinterest. If the group had contained one RPG enthusiast it may have been enough to trigger curiosity in the others just as watching others play had resulted in Buffy attaining last minute popularity. In this case, however, these games were unfamiliar and as a result they simply failed to register with these users.

This suggests that while preferences are open to alteration, there needs to be a motivating catalyst or transitional support when players are confronted by a complex and unfamiliar genre. Such a catalyst might be social - but it is also possible that it could be textual, or trans-textual: had these RPG's been set in 'Harry Potter world', for instance, it is likely that the girls would have at least tried them, regardless of their unfamiliarity with the genre.

\section{CONCLUSION}

So, what computer games do girls like? The answer is that it depends. Socialization may well play its part, yet our gaming preferences will also depend on where we are, what we know, who we know, what we've tried, and what we've grown tired of. Distinctions in taste between male and female players reflect patterns in games access and consumption that spring from (very) gendered cultural and social practices. As this suggests, accounts of gaming preference need to be situated within a framework that incorporates reference to players' previous access to games and existing gaming knowledge. Gaming preferences need to be conceptualized within a paradigm that can accommodate mobility, increment, learning and alteration.

Different people will accumulate particular gaming skills, knowledge and frames of reference, according to the patterns of access and peer culture they encounter - and these accumulations will pool as predispositions, and manifest as preferences. Familiarity and competence feed into a player's experiences of gaming, partly determining the pleasures that he or she will expect, recognize and access, and thereby impacting on preferences that might be expressed as a result. Preferences are an assemblage, made up of past access and positive experiences, and subject to situation and context. The constituents of preference (such as access) are shaped by gender and, as a result, gaming preferences manifest along gendered lines. It is not difficult to generate data that will indicate that gendered tastes exist, but it is short sighted to divorce such preferences from the various practices that form them. To attribute gaming tastes directly, solely or primarily to an individual subjects' gender, is to risk underestimating the complexities of both subjectivity and preference. 
On a final note, it is absolutely possible that various factors bolstered the club members' enthusiastic engagement with games during the computer gaming sessions described in this paper. It could be argued that we created something of an artificial environment, an anomalous 'bubble' where it was the norm that girls and women enjoy playing and talking about computer games. However is also true that outside of any such bubble, the relationship of girls - or boys, women or men - to computer games, is just as constructed.

\section{ACKNOWLEDGEMENTS}

This paper is abridged from "Contexts, Gaming Pleasures and Gendered Preference" - an article that is presently under review with the journal Simulation and Gaming (symposium issue on video game theory, D. Myers and J. Colwell, eds.). This abridgement appears here with the editors' permission. This research was undertaken with the support of the Eduserv Foundation (www.eduserv.org.uk) as part of a project on computer games, motivation and gender. Pplease see http://www.lkl.ac.uk/research/carr.html for further information. I would also like to thank my colleague Caroline Pelletier who was the primary facilitator of these gaming sessions.

\section{REFERENCES}

1. Bryce, J. and Rutter, J. "Killing Like a Girl: Gendered Gaming and Girl Gamers' Visability”. Paper presented at the Computer Games and Digital Culture Conferences, Tampere, Finland 2002.

2. Buckingham, D. and Sefton-Green, J. Cultural Studies Goes to School: Reading and Teaching Popular Media. London: Taylor and Francis. 1994

3. Cassell, J. and Jenkins, H. From Barbie to Mortal Combat: Gender and Computer Games. Cambridge, Mass.: MIT Press. 2000

4. Jenkins, H. Textual Poachers, Television Fans and Participatory Culture. New York: Routledge 1992

5. Kerr, A. "Women Just Want to Have Fun: A Study of Adult Female Players of Digital Games". Paper presented at the Level Up: Digital Games Research Conference, Utrecht 2003

6. Krotoski, A. Chicks and Joysticks: An Exploration of Women and Gaming: Entertainment and Leisure Software Publishers Association (ELSPA) 2004

7. Schott, G. and Horrell, K. "Girl Gamers and their Relationship with the Gaming Culture". Convergence, 6, 2000 pp 36-53 